\title{
Charge transport in conjugated polymer-semiconductor nanoparticle composite near the percolation threshold
}

\author{
L. S. Cardoso ${ }^{1}$ - G. E. Gonçalves ${ }^{2,3}$ - D. H. F. Kanda ${ }^{4}$ R. F. Bianchi ${ }^{3} \cdot$ H. N. Nagashima ${ }^{4}$
}

Received: 29 September 2017 / Accepted: 21 November 2017 / Published online: 25 November 2017

c) Springer-Verlag GmbH Germany, part of Springer Nature 2017

\begin{abstract}
This paper describes a new statistical model to predict the frequency dependence of the conductivity of conjugated polymersemiconductor nanoparticle composites. The model considers AC conduction in an inhomogeneous medium represented by a two-dimensional model of resistor network. The conductivity between two neighboring sites in the polymer matrix and the semiconductor particles is assumed to obey the random free energy barrier model and the Drude model, respectively. The real and imaginary parts of the AC conductivity were determined using the transfer-matrix technique, and the statistical model was applied to experimental data of thin films composed of polyaniline (PANI) and indium-tin-oxide (ITO) nanoparticles. The conductivity critical exponent $(s)$ obtained in two dimensions for PANI/ITO films below the percolation threshold was found to be 2.7, which is greater than the universal value of $s$ described by the classical percolation theory $(s=1.3)$. This non-universality is explained by the existence of a local electric field distribution in the bulk of the nanocomposite. Finally, these results are discussed in terms of the distribution of potential barriers that vary according to the concentration of ITO amount in the composite.
\end{abstract}

\section{Introduction}

Recently, polymer nanocomposites have attracted much attention in the academic area due to their fascinating potential for technological applications in many fields such as sensors, light-emitting diodes and super capacitors [1-9]. Polymer nanocomposites consist of a polymer matrix filled with inorganic/organic nanoscale additives. In particular, these materials have attracted a considerable research interest because they have exhibited better mechanical, electrical, chemical and optical properties than their counterparts $[10,11]$. Polymer matrices filled with inorganic nanoparticles combine the features of the polymer matrices such

\section{S. Cardoso}

licardoso@ifsc.usp.br

1 Instituto de Física de São Carlos, Universidade de São Paulo, São Carlos, SP 13560-970, Brazil

2 Instituto Federal Minas Gerais, IFMG-Campus Ouro Preto, Ouro Prêto, MG 35400-000, Brazil

3 Departamento de Física, Universidade Federal de Ouro Preto-UFOP, Ouro Prêto, MG 35400-000, Brazil

4 Departamento de Física e Química, Universidade Estadual Paulista, Campus de Ilha Solteira, Ilha Solteira, SP 15385-000, Brazil as low weight and flexibility with the unique characters of the inorganic nanoparticles. Over the past of few decades, many experimentalist and computer scientists are trying to understand the relationships between the interactions, phase behavior and morphology of polymer nanocomposites [12-15]. These studies have revealed that the macroscopy properties of the polymer nanocomposites depend strongly of the morphology, especially of the microscopy morphology of the nanoscale additives in the polymer matrix.

Furthermore, many studies have demonstrated that the superior performance of the polymer nanocomposites compared to those made of conventional composites can be attributed to their much larger exposed surface area and the very short distance between the nanoparticles [16-18], which provide access to peculiar morphologies, such as interpenetrating networks, even at very low concentrations of dispersed nanoparticles [19, 20]. Consequently, the electrical properties of polymer nanocomposite films can be dominated by nanoparticles in some ranges of concentration, and then by the percolation threshold of these systems [21-23]. The percolation threshold in the classical percolation theory is defined as the nanoparticle amount necessary to form a continuous conductive path by physical contact of the nanoparticles. 
Several conduction mechanisms can be involved in the charge transport process of polymer nanocomposites and its investigation remains a challenge due the complex microstructure of these systems. The nanoparticle/nanoparticle interaction, polymer matrix/polymer matrix interaction and nanoparticle/polymer matrix interaction play an important role in the conduction mechanisms and they should be taken in consideration for a deeper understanding of the electrical properties of these materials.

Based on this, we developed a new statistical model of resistor network that generates the morphological structure of polymer nanocomposites and calculate the alternating electrical conductivity using a transfer-matrix technique $[24,25]$. The model was applied to simulate the virtual microstructure of polyaniline (PANI) and indium-tin-oxide (ITO) polymer nanocomposites and to reproduce the real and imaginary parts of the AC conductivity of these films. ITO and PANI are materials used as transparent anodes for organic light-emitting displays [26-28], and the recent combination of ITO/PANI nanocomposites has now generated a new field for the development of printed, organic, and flexible transparent electrodes. The model takes into account the disordered structure of PANI, the random distribution of nanoparticles, and the aggregates of ITO in the polymeric matrix. The impedance between two neighboring sites of the polymeric matrix was calculated for PANI using the Random Free Energy Barrier Model [29], while the impedance between two neighboring sites from the conductive phase was calculated by the Drude model [30].

\section{Theory, film preparation and statistical model}

\subsection{Percolation theory and critical exponent of conductivity}

The electrical conductivity behavior of polymer nanocomposites has been commonly described by the percolation theory [31]. It is widely known that the DC electrical conductivity of polymer nanocomposites depends of the nanoparticles concentration. For polymer nanocomposites with low content of nanoparticles, the conductivity is determined by the polymer matrix as result of the large mean distance between the nanoparticles. Increasing the conducting phase content, the nanoparticles get closer and at a critical point of content, known as percolation threshold, a continuous conductive path is formed throughout the polymer matrix by the physical contact of the nanoparticles and then the electrical conductivity is dominated by these nanoparticles. In the classical percolation theory, a continuous network must be present in a nanocomposite through the connection between adjacent nanoparticles [31]. In this case, the electrical conductivity $(\sigma)$ of the nanocomposite may depend not only on the percolation threshold concentration $\left(p_{c}\right)$ but also on the conductive component concentration $(p)$ and the conductivity critical exponent above $\left(p>p_{c}\right)$ the percolation threshold $(t)$ as in the following equation:

$\sigma \sim\left(p-p_{c}\right)^{t}$.

The exponent $t$ in Eq. (1) is associated with different conduction mechanisms involved in electronic transport phenomena [32-34]. However, for $p_{c}>p$, the average distance between adjacent nanoparticles prevents the formation of a percolating cluster. The conductivity is then governed by the narrow gaps between the neighboring nanoparticles. As $p$ increases and approaches $p_{c}$, the conductivity of the composite can be described by the following equation:

$\sigma \sim\left(p_{c}-p\right)^{-s}$,

where $s$ is the conductivity critical exponent below the percolation threshold. Finally, the critical exponent $s$ and the exponent $t$ are considered universal in the theory of classical percolation and vary according to dimensionality $d$. In this case, $s$ assumes values from 1.1 to 1.3 for $d=2$, and from 0.7 to 1.0 for $d=3$, while the critical exponent $t$ assume values from 1.1 to 1.3 for $d=2$, and 1.6 to 2.0 for $d=3$ [35]. Moreover, it is well known that conventional conducting composites prepared with conductive particles dispersed in an insulating matrix show the percolation transition. The universal behavior of critical exponents of electrical conductivity has been experimentally found in a limited number of experiments on real disordered composites and theoretically found for a random resistor network model [36]. The change from insulating to conducting state occurs when the conductive particles or cluster are in contact with each other and form a continuous percolating path throughout the matrix (geometrical connectivity). On the other hand, and in contrast with the classical percolation theory, non-universal behavior by the critical exponents of electrical conductivity has been observed in percolative composites by several authors [37-39]. In these cases, the authors have demonstrated that the conductive particles are connected not geometrically, but electrically by tunneling mechanism. Thus, the percolation occurs even in the absence of a geometrically connected network [40, 41]. Consequently, the study of the behavior of non-universal critical exponents of conductivity associated with impedance measurements is an important tool to analyze the processes of electrical conduction responsible for the transition from the insulating to conducting phase.

\subsection{Preparation and characterization of PANI/ITO nanocomposites}

Polyaniline (PANI) was synthesized as described elsewhere $[42,43]$. PANI/ITO hybrid material was obtained by the 
addition of ITO nanoparticles purchased from SigmaAldrich (diameter $<50 \mathrm{~nm}$ ) to a solution of PANI in NMP ( $N$-methylpyrrolidone). The PANI/ITO films were obtained by a casting method to produce $4,8,12,16$, and $20 \%$ of ITO by weight of film.

The AC conductivity of the PANI/ITO films was measured by impedance spectroscopy using a Solartron 1260 by Impedance/Gain Phase Analyzer in the frequency range from $10^{1}$ to $10^{6} \mathrm{~Hz}$, whereas DC conductivity was obtained using the two-probe method. The equipment uncertainty of the impedance measurement was smaller than $1 \%$, and a $5 \%$ error bar was introduced to allow for uncertainty about film thickness and the exact area of the top electrode.

\subsection{Statistical model}

A statistical model of resistor network was developed in this work to study the electrical transport properties of ITO/ PANI films. The model developed in Fortran 90 takes into account the electron hopping and tunneling mechanisms through the dispersed nanoparticles and aggregates of ITO in the PANI matrix, as well as the disordered arrangement of the polymer chains in the matrix and the interfacial processes between the conducting phase and polymeric matrix. Consequently, the theoretical model introduced in this paper focuses on two important aspects of the material: its geometrical structure and electrical conductivity. The statistical model computes the conductance per unit length of networks consisting of very long two-dimensional strips built by juxtaposing $N$ square cells. Each cell is formed by polymer chains consisting of a sequence of resistors in series arranged randomly, and by particles and aggregates consisting of resistors connected to the nearest neighbors of the central site, also distributed randomly in the cell. The impedance values of these resistors vary according to the structure and characteristics of the material. They represent the difficulty of the charge transfer processes of electronic carriers and can be represented by a distribution of energy barriers [44]. Finally, the calculation of the complex conductivity of the network is performed by applying a transfer-matrix technique $[24,45]$, which enables the calculation of DC conductivity for a random resistor network. As a result, the model generates the structure of the ITO nanoparticles, which are randomly distributed in the square cell. Nanoparticle size is determined by the choice of diameter, in a first approximation taken from a Gaussian distribution probability [46] centered in $d_{0}$ with dispersion $\Delta d$. An empty lattice site $m$ of the two-dimensional cell is randomly occupied, representing the center of a nanoparticle. All neighboring sites located within a radius of $d_{0} / 2$ are then connected by a resistor with $Z_{\text {ITO }}$ impedance. A search of all of its sites is subsequently performed to look for a pair of nearest neighbor sites that does not belong to the same nanoparticles. If such a pair is found, then these sites are connected, with probability $p_{h}$, by a resistor of the same impedance. The idea is to incorporate hopping and tunneling mechanisms. The process is repeated until the chosen bond density of ITO is reached.

The molecular chains are then built to generate the polymeric matrix. Initially, an empty site $m$ of the cell is randomly chosen and connected by a resistor with impedance $Z_{\text {PANI }}$ to an empty nearest neighbor site $n$, also randomly chosen. The process is repeated until the chosen polymer chain size is reached. A search in all of its sites is subsequently performed to look for a pair of nearest neighbor sites not connected by a resistor, which may or may not belong to the same chain. If such a pair is found, then these sites are connected, with probability hopping $p_{h}$, by a resistor with $Z_{\text {PANI }}$ impedance. The idea is to incorporate induced charges and a hopping mechanism. By repeating the procedure described above, new polymer chains are built inside the cell until a determinate concentration $k$ of the occupied lattice bonds is reached.

The complex impedance $Z_{\text {Iто }}$ between two sites of nanoparticles is represented by Drude's model [30], proposed by Drude in 1900, and is applied to materials that have delocalized wave functions. In our model, each resistor representing the bonds from ITO nanoparticles is assigned an impedance value in accordance with the following equation:

$Z_{\text {ITO }}=A\left(\frac{1-i \omega \tau}{\tau}\right)$,

where $\tau$ corresponds to the waiting time, $\omega$ is the angular frequency of the electric field and $A$ is a constant that is a function of carrier density, charge electron, electron mass, and the distance between sites.

Hopping conduction is the predominant transport mechanism in an insulating matrix due to the strongly localized electrons. The random free energy barrier model (RFEB) was proposed by Dyre to explain conduction in disordered media. According to this model, the charge carrier must overcome the energy barriers randomly distributed between a minimum $E_{\min }$ and a maximum energy $E_{\max }$, within an equally probable distribution of free energy barriers.

In the regime of low frequencies, the charge carriers have sufficient time to meet high-energy barriers; consequently, the lowest jumping frequency $\gamma_{\min }$ dominates the process. Therefore, a charge carrier can jump from one site to its neighboring empty site with a minimum frequency given by the following equation:

$\gamma_{\min }=\gamma_{0} \exp \left(-E_{\max } / k T\right)$,

where $\gamma_{\mathrm{o}}$ is the escape frequency, $k$ the Boltzmann constant, and $T$ the absolute temperature. In this regime, the real component of the conductivity is independent of frequency. However, the original RFEB model takes into account only the electronic conduction [29], so that as proposed by 
Bianchi et al. [47] for a better understanding of the phenomena involved in conducting of the material-the term that refers to the dielectric contribution $\left(i \omega \varepsilon^{\prime}\right)$ must be added to the equation proposed by Dyre. Therefore, the impedance $Z_{\text {PANI }}(\omega)$ between two adjacent insulating sites, as a function of the angular frequency $\omega$, can be written as follows:

$Z_{\mathrm{PANI}}=\left\{K\left[-i \omega+\frac{i \omega \ln \left(\gamma_{\max } / \gamma_{\min }\right)}{\ln \left[\frac{1+\left(i \omega / \gamma_{\min }\right)}{1+\left(i \omega / \gamma_{\max }\right)}\right]}\right]+i \omega \varepsilon^{\prime}\right\}^{-1}$,

where $\gamma_{\min }$ is the minimum frequency of jumps and $\gamma_{\max }$ is the maximum frequency of jumps, $\omega$ is the angular frequency of the electric field, and $K$ is a constant that depends on the density of charge carriers, the electron charge, the carrier effective mass, the waiting time, and distance between sites. The electrical permittivity of the medium is given by $\varepsilon^{\prime}=k \varepsilon_{0}$, where $k$ is the dielectric constant and $\varepsilon_{0}$ is the vacuum permittivity.

The impedance $Z_{\mathrm{INT}}$ at the interface between the insulating and conducting phase is given as follows:

$Z_{\mathrm{INT}}=\left(Y_{\mathrm{PANI}}+Y_{\mathrm{ITO}}\right) / A Y_{\mathrm{PANI}} Y_{\mathrm{ITO}}$,

where $Y_{\text {PANI }}$ and $Y_{\text {ITO }}$ are the admittances of the PANI and ITO, respectively. The coefficient $A=2$ is employed for the square lattice [48]. Thus, replacing $Y_{\mathrm{PANI}}=1 / Z_{\mathrm{PANI}}$ and $Y_{\text {ITO }}=1 / Z_{\text {ITO }}$, the impedance $Z_{\text {INT }}$ can be rewritten as follows:

$Z_{\mathrm{INT}}=\left(Z_{\mathrm{PANI}}+Z_{\mathrm{ITO}}\right) / 2$.

The impedance provided by Eqs. (3), (5), and (7) are used to calculate the conductivity of a square cell using the technique of the transfer matrix. This cell consists of a resistor network simulating the structure of the nanocomposite. A schema from the structure of the statistical model for the nanocomposite PANI/ITO is shown in Fig. 1, which demonstrates the three kinds of impedance: $Z_{\mathrm{INT}}, Z_{\mathrm{PANI}}$, and $Z_{\mathrm{ITO}}$.

After construction of the first cell, a new cell is then built beside the first cell. Of course, care should be taken when

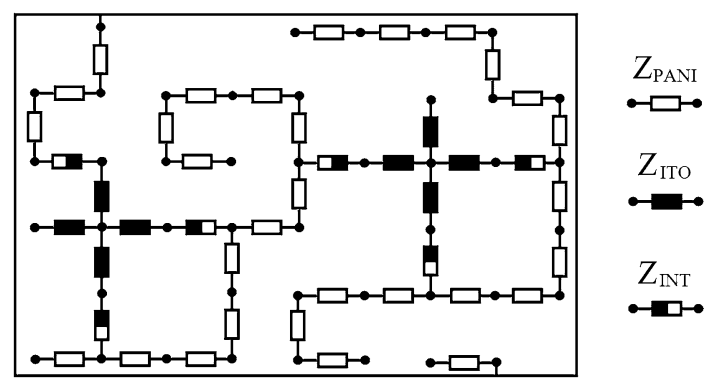

Fig. 1 Model for PANI/ITO composite based on three elements, two of which represent the constituent phases and one the interface passing from one cell to another: horizontal and vertical bonds on the boundary must be stored to correctly construct the next cell. This process is repeated until an infinitely long strip has been generated. A sample of nanocomposite thin film can be represented by a long strip formed by juxtaposing $N$ square cells with $L \times L$ sites.

\subsubsection{Determination of electrical conductivity}

Derrida et al. [45] introduced a transfer-matrix formulation for computing the conductance of random resistor networks. The matrix $A_{L}$ represents the conductance of the very long strip with resistors placed at random on a square lattice. Each time a new resistor is added, the matrix $A$ is modified. Derrida and colleagues studied the case in which the resistors are either cut with probability $(1-p)$ or present with probability $p$.

When one adds a horizontal resistor $R$ onto a site $\alpha$ of network, then the matrix $A_{m n}$ becomes a new matrix $A^{\prime}{ }_{m n}$, according to the following equation:

$A_{m n}^{\prime}=A_{m n}-\frac{R \cdot A_{m \alpha} A_{\alpha m}}{1+R \cdot A_{\alpha \alpha}}$,

where $R$ is the resistance of resistor.

The effect of the vertical bonds is given by:

$A_{m n}^{\prime \prime}=A_{m n}^{\prime}+\frac{1}{R}\left(\delta_{\alpha, m}-\delta_{\beta, m}\right)\left(\delta_{\alpha, n}-\delta_{\beta, n}\right)$,

where $\delta_{m n}$ is Kronecker's symbol ( $\delta_{m n}=1$ if $m=n$ and $\delta_{m n}=0$ otherwise). The conductivity per unit length $\sigma$ of a strip of length $L$ is given by:

$\sigma=\lim _{L \rightarrow \infty} A_{11} / L$,

where $A_{11}$ is an element at the first row and column of the matrix for $L \rightarrow \infty$.

We extend this transfer-matrix technique to calculate the $\mathrm{AC}$ conductivity of a resistor network, introducing the impedance $Z$ in place of the resistance $R$. Impedance that represents the difficulty of charge carrier jump from one site to another (potential barrier) varies according to the structure and characteristics of the material. For PANI/ITO films, we use the Drude model to represent the impedance of ITO and the Dyre model to represent the impedance of PANI.

\section{Results}

The DC conductivity behaviors of the PANI/ITO nanocomposites with increasing concentration of ITO nanoparticles can be observed in the DC electrical conductivity measurements shown in Fig. 2. Here, it is observed that the conductivity of PANI/ITO nanocomposites shows a substantial 


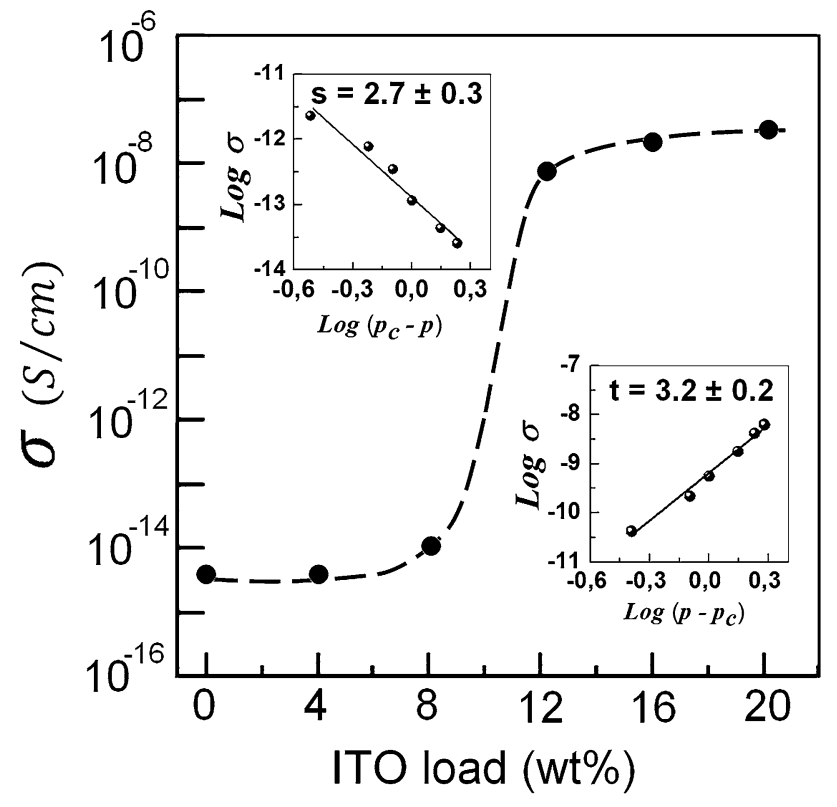

Fig. 2 Electrical conductivity of PANI/ITO composites as a function of ITO load (wt \%)

increase of about six orders of magnitude in the percolation region located between the concentrations of 8 and $12 \mathrm{wt} \%$, suggesting that the percolation threshold is $10.6 \mathrm{wt} \%$ of ITO.

The values of the critical exponents of electrical conductivity obtained for the PANI/ITO nanocomposites, below and above the percolation threshold $\left(p_{c}=0.106\right)$, were calculated from the determination of the angular coefficient obtained by linear fit of the conductivity $\sigma$ as a function of $\left(p_{c}-p\right)$ and $\left(p-p_{c}\right)$. The values obtained for the critical exponent were $t=3.2$ and $s=2.7$, which were greater than the values expected based on classical percolation theory.

The code developed in Fortran 90 generates the morphological structure of PANI/ITO nanocomposites, taking into account the highly disordered structure of weakly doped PANI structure, the homogeneous dispersion of nanoparticles of ITO, and the formation of aggregates. An example of a cell formed by $80 \times 80$ sites for PANI/ITO (88/12) is shown in Fig. 3. A sample of nanocomposite thin film can be represented by a long strip formed by juxtaposing $N$ square cells with $L \times L$ sites. The ITO nanoparticles are represented by a dark line and the polyaniline chains are represented by a gray line.

The real and imaginary components of AC conductivity measurement, as well as the results of the simulation, are presented in Fig. 4. In the simulation, a strip of 1000 square cells was used with $50 \times 50$ sites. The measurement was faithfully reproduced by our model. The free charge response of ITO was modeled using the Drude model, while the motion of charge carriers in the polymer matrix was modeled by Dyre's RFEB model.

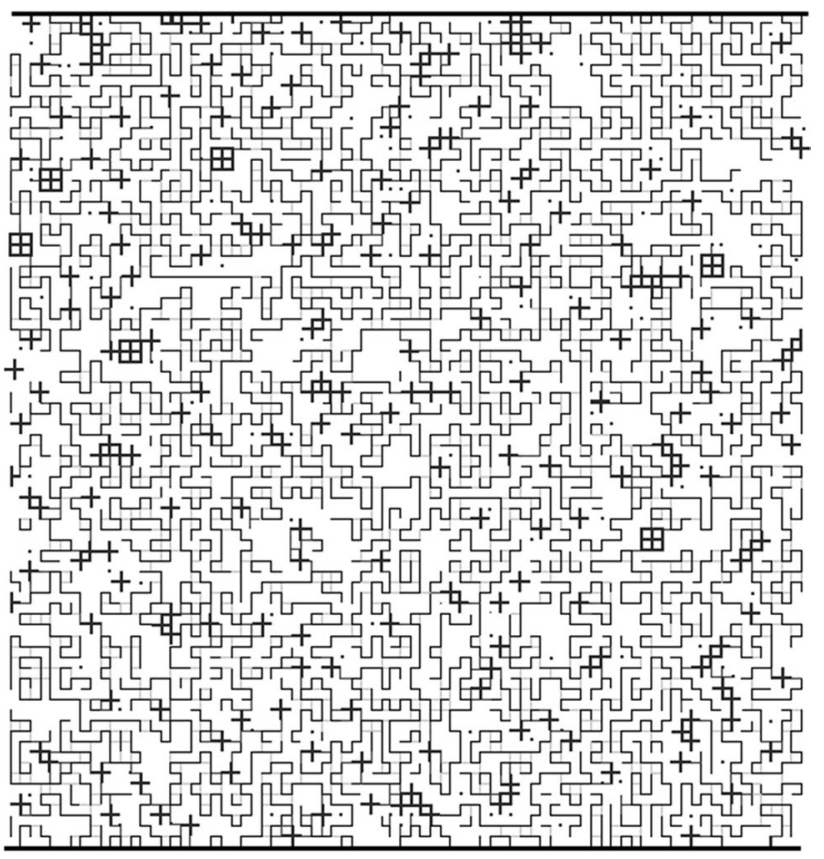

Fig. 3 Example of a cell formed by $80 \times 80$ sites for PANI/ITO $(88 / 12)$

The statistical model parameters used to fit the experimental data for PANI/ITO nanocomposites are presented in Table 1 . The Dyre constant $K$, the minimum jumping frequency $\gamma_{\min }$, and maximum jumping frequency $\gamma_{\max }$ are related to the Dyre model. Drude's constant $A$ and the waiting time of the charge carriers $\tau$ are related to the Drude

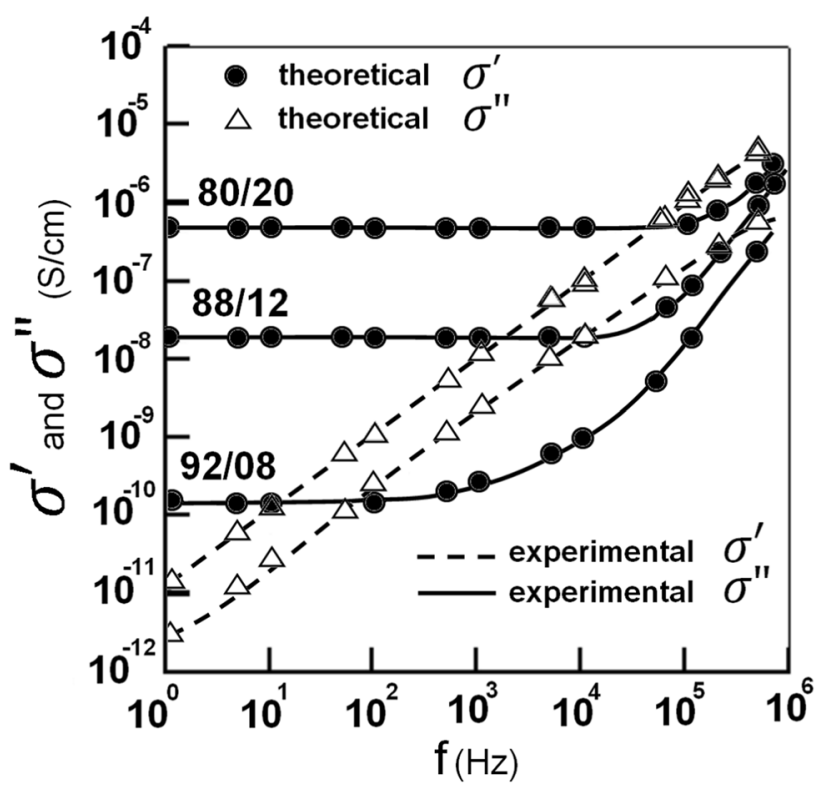

Fig. 4 Simulation and experimental measurements of complex conductivity of the PANI/ITO nanocomposites as a function of frequency with concentrations of 08,12 , and 20 at $\%$ ITO 
Table 1 Parameters obtained from the complex conductivity plots observed for PANI/ITO nanocomposite

\begin{tabular}{llllll}
\hline $\begin{array}{l}\text { ITO } \\
\text { loading } \\
(\mathrm{wt} \%)\end{array}$ & $K(\Omega \mathrm{s})^{-1}$ & $\begin{array}{l}\gamma_{\min } \\
(\mathrm{rad} / \mathrm{s})\end{array}$ & $\begin{array}{l}\gamma_{\max } \\
(\mathrm{rad} / \mathrm{s})\end{array}$ & $A(\Omega \mathrm{s})$ & $\tau(\mathrm{s})$ \\
\hline 1 & $3.6 \times 10^{-12}$ & $4.0 \times 10^{1}$ & $1.0 \times 10^{12}$ & $4.0 \times 10^{7}$ & $8 \times 10^{-13}$ \\
5 & $3.7 \times 10^{-12}$ & $5.0 \times 10^{1}$ & $1.0 \times 10^{12}$ & $4.0 \times 10^{7}$ & $4.0 \times 10^{-13}$ \\
8 & $3.8 \times 10^{-12}$ & $1.5 \times 10^{2}$ & $1.0 \times 10^{12}$ & $4.0 \times 10^{7}$ & $3.0 \times 10^{-13}$ \\
12 & $4.2 \times 10^{-12}$ & $2.0 \times 10^{4}$ & $1.0 \times 10^{12}$ & $4.0 \times 10^{7}$ & $2.0 \times 10^{-13}$ \\
20 & $1.0 \times 10^{-11}$ & $1.0 \times 10^{5}$ & $1.0 \times 10^{12}$ & $4.0 \times 10^{7}$ & $1.0 \times 10^{-13}$ \\
\hline
\end{tabular}

Source: elaborated by the authors

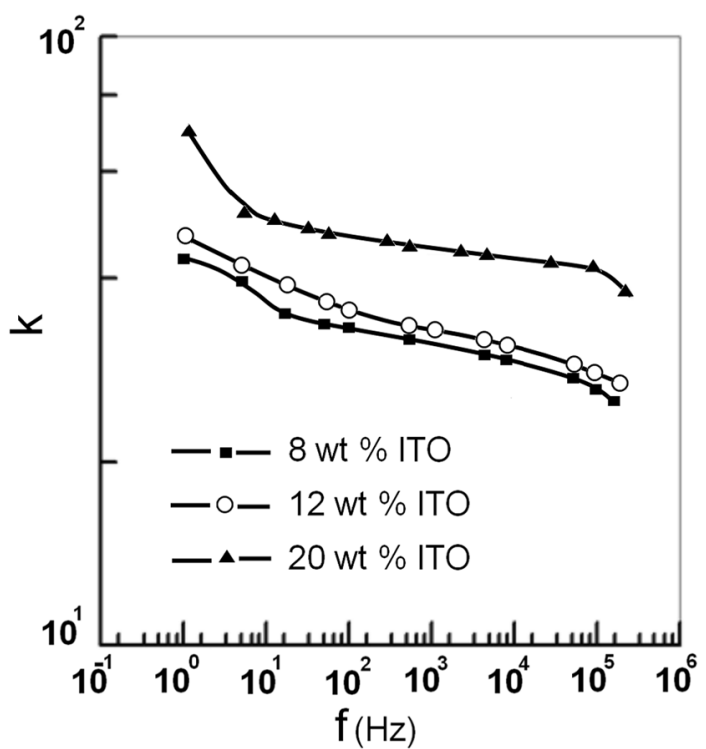

Fig. 5 Behavior of the dielectric constant in PANI/ITO composites as a function of the frequency

model. The dielectric constants, used as fitting parameters, are presented in Fig. 5. It is observed that the most sensitive parameters are the Dyre constant and minimum jumping frequency. A $10 \%$ change in the $K$ and $\gamma_{\min }$ parameters resulted in 10 and $9 \%$ changes in the real conductivity, respectively. A $10 \%$ change in the $\gamma_{\max }, A$, and $\tau$ parameters resulted in a $0.7 \%$ change in the real conductivity, while for the ITO loading it resulted in a $3 \%$ change in the real conductivity.

The value of the Drude constant was $A=4.0 \times 10^{7} \Omega$ s for all PANI/ITO nanocomposite samples. In the Dyre RFEB model, hopping is the dominant conduction mechanism for the electron transport occurring in a disordered medium in the presence of free energy barriers between the trapping sites. The energy barriers are randomly distributed between a minimum value $E_{\min }$ and a maximum value $E_{\max }$, which results in two limiting carrier escape frequencies, $\gamma_{\max }$ and $\gamma_{\min }$, respectively. The maximum value frequency $\gamma_{\max }=10^{12} \mathrm{rad} / \mathrm{s}$ was the same for all samples.
The dielectric constant $k$ showed a weak variation with frequency. The expected decrease in $k$ with increasing frequency, which occurs as the interfacial polarization that dominates in the low-frequency region, gradually diminishes toward high frequencies. The dielectric constant increased with ITO nanoparticle content at all the frequency ranges.

\section{Discussion}

The electrical conductivity obeys the power law according to Equations (1) and (2), where $t$ and $s$ are the conductivity critical exponents determined above and below the percolation threshold, respectively. These exponents are considered universal in classical percolation theory in which the conductivity is established through the direct physical contact between the adjacent nanoparticles (geometric percolation). Below the percolation threshold, the conductivity of the nanocomposites remains close to that of the insulating polymer matrix.

The values of the critical exponents of electrical conductivity obtained for the PANI/ITO nanocomposites were $t=3.2$ and $s=1.7$. These values are greater than the expected values for classical percolation theory. This non-universality can be explained by the tunneling-percolation model, which takes into account the tunneling of electrons between two adjacent conductive particles $[38,49]$. In this case, an electrical percolation occurs when "current flows" through the system, that is, when a continuous conductive path between the electrodes is formed, even in the absence of a geometrically connected network.

Balberg and colleagues $[50,51]$ have studied percolation and tunneling in composite materials by capacitance probe microscopy. They proposed a model in which the formation of a network of electrical percolation through the tunneling mechanism is considered. This mechanism is characterized by a distribution of values of resistances that vary according to the average distance between the conductive particles. In a resistor network model, the value of the critical exponent depends not only on the concentration of these resistors on the network, as predicted by the percolation theory, but also on the distribution of resistance values assigned to these resistors.

The real and imaginary components of AC conductivity measurement for the PANI/ITO nanocomposite, as well as the results of the simulation, are presented in Fig. 4 and in Table 1. The parameter that stands out in Table 1 is that of the minimum jumping frequency $\gamma_{\min }$, which represents the frequency at which the AC conductivity becomes dependent on frequency and which is related to the higher energy barriers present in the bulk of the sample.

The behaviors of the minimum jumping frequency $\gamma_{\min }$ as a function of the ITO content in PANI/ITO nanocomposites 
are shown in Fig. 6. The increase in $\gamma_{\min }$ by three orders of magnitude in the region of the percolation threshold can be related to the decrease in energy barriers present in the thin layers of the polymeric matrix located between adjacent ITO aggregates. These structures form small capacitors where the local electric field can be sufficiently intense to allow charge transport by hopping or tunneling.

Simoes $[52,53]$ observed an anomalous percolative behavior in carbon nanotube composites. These composites show lower electrical resistance and higher dielectric constant with increases in the conductive filler below the percolation threshold. This can be explained as a consequence of the formation of capacitor networks in the bulk of the composites. The capacitance of the local capacitors is calculated by taking into account the distance between the neighboring carbon nanotubes, as well as their relative positions. As a consequence, the statistical model developed in this work applies the modified equation of Dyre, expressed by Eq. (5), to represent the conducting mechanism of charge carriers between adjacent sites of the polymer matrix. The behavior of the minimum jumping frequency $\gamma_{\min }$, which is associated with the heights of the highest potential barriers, indicated an increase in conductance in the matrix regions of PANI with increasing content of conductive particles. This can be explained by taking into account the increased local electric field in the interior of small capacitors.

As the content of ITO nanoparticles increases, the number of conductive paths increases. Thus, the average distance between the conductive particles becomes smaller. The structure of the nanocomposite ITO before the percolation threshold is shown in Fig. 7. In this case, the conductor paths do not allow connection between the electrodes.

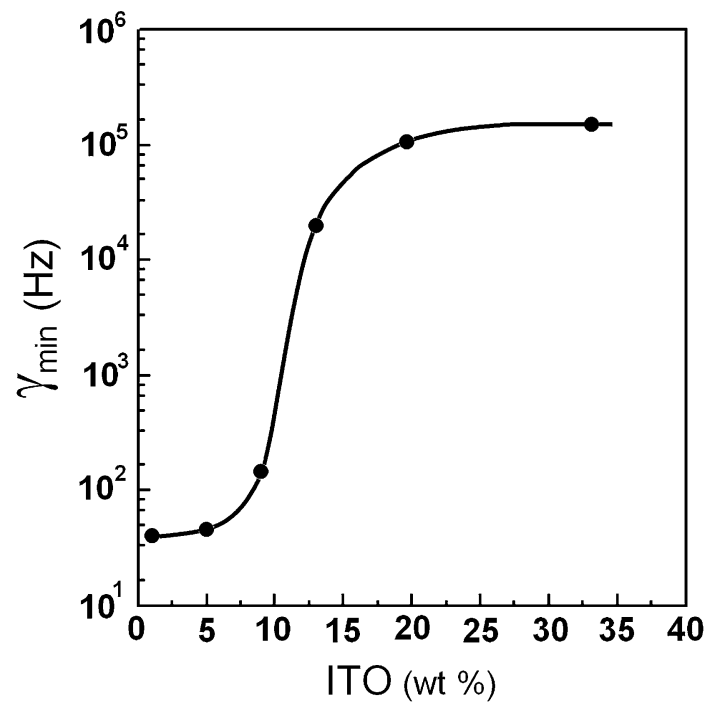

Fig. 6 Minimum jumping frequency $\gamma_{\min }$ as a function of the ITO content observed in PANI/ITO composites

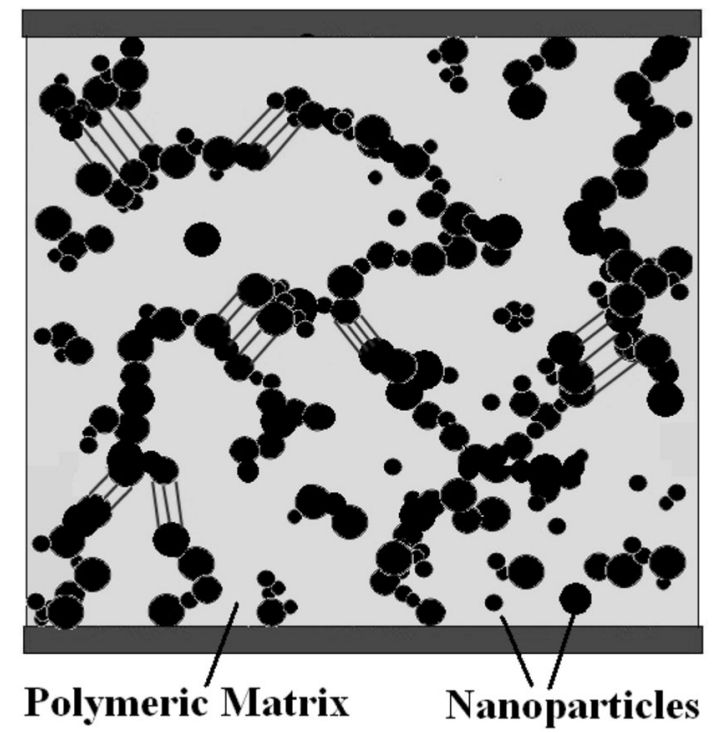

Fig. 7 Schematic representation of conductive paths of ITO nanoparticles, considered before the percolation threshold is reached; parallel segments represent the regions in the matrix where the electric field is intense

The structure of nanocomposite before the percolation threshold is also shown in Fig. 7. In this case, the conductive paths formed by ITO aggregates do not allow physical contact between the electrodes. The conductive nanoparticles (represented by black circles) are randomly dispersed in the polymer matrix. The circuit formed by metallic wires and small capacitors, as shown in Fig. 8, is an equivalent circuit of the structure shown in Fig. 7. This circuit represents the conductive paths and the charges

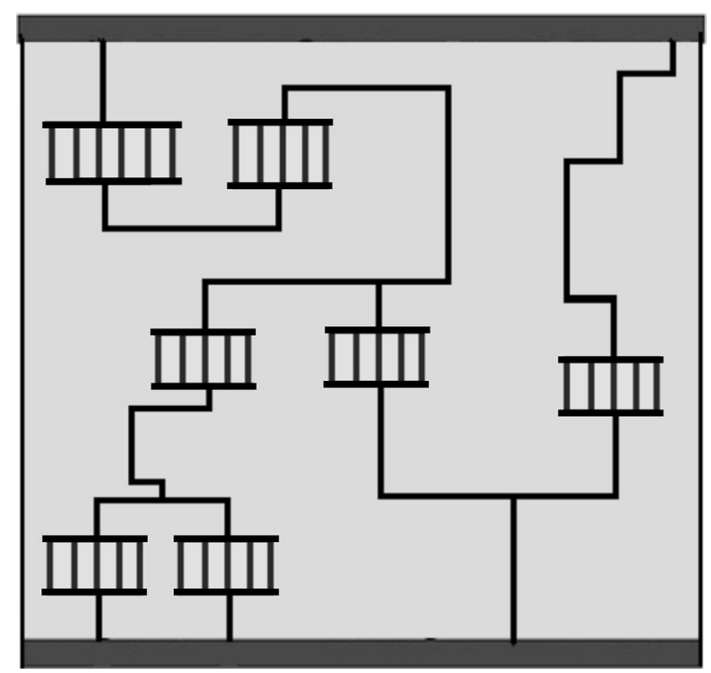

Fig. 8 Black segments represent the paths formed by particles of ITO; capacitors represent regions of interface where the electric field is more intense 
accumulated in certain regions in the bulk of the sample. The electrical field is illustrated by parallel segments between the capacitor plates.

The potential difference applied to samples of the PANI/ ITO nanocomposites is divided between the capacitors that are present along a path that connects the two electrodes. Thus, a distribution of potential differences will occur in the bulk of the sample. Since the distance between the plates is of the same order of magnitude as the conductive particle aggregates, the result is a significant increase in the local electric field intensity and the number of charge carriers at the interfaces between the phase conductor and polymeric matrix. The increased electric field intensity decreases the effective height of the potential barriers due to the Poole-Frenkel effect $[54,55]$ which favors the hopping and tunneling conduction mechanism.

\section{Conclusions}

We introduced a two-dimensional statistical model that was developed based on resistor networks that reproduce the morphological structure and that calculate the alternating conductivity of a PANI/ITO nanocomposite. The calculation of the conductivity was carried out using the modified Dyre's model in the polymer matrix (PANI) region and Drude's model in the nanoparticles and aggregates of ITO. The simulations reproduced the alternating conductivity measurements in samples in a large range of frequencies and explained the conduction mechanism near the percolation threshold that give rise to the conductivity critical exponents larger than those provided by the classical percolation theory.

Near the percolation threshold, the PANI/ITO nanocomposite becomes less resistive before the physical contacts between ITO nanoparticles have been reached, due to the accumulation of charge carriers at the interfaces and the occurrence of hopping and tunneling mechanisms in the regions of high intensity of local electric field, which are randomly distributed in the bulk of the material. This distribution of values of local electric field, which vary according to the average distance between the conductive nanoparticles, clarifies the behaviors of the minimum jumping frequency $\gamma_{\min }$ and the increase in dielectric constant $k$ in the polymeric matrix in response to increases in the concentration of nanoparticles in the composite.

Acknowledgements This work was supported by FAPESP, FAPEMIG, $\mathrm{CNPq}, \mathrm{CAPES}$, and the National Institute of Electronic Organics (INEO/MCT/CNPq/FAPESP).

\section{References}

1. L. Xue, W. Wang, Y. Guo, G. Liu, P. Wan, Sensors Actuators B Chem. 244, 47 (2017)

2. T. Sen, S. Mishra, N.G. Shimpi, RSC Adv. 6, 42196 (2016)

3. S. Abdulla, T.L. Mathew, B. Pullithadathil, Sensors Actuators B Chem. 221, 1523 (2015)

4. H.-K. Seo, H. Kim, J. Lee, M.-H. Park, S.-H. Jeong, Y.-H. Kim, S.-J. Kwon, T.-H. Han, S. Yoo, T.-W. Lee, Adv. Mater. 29, 1605587 (2017)

5. H.J. Yun, S.J. Kim, J.H. Hwang, Y.S. Shim, S.-G. Jung, Y.W. Park, B.-K. Ju, Sci. Rep. 6, 34150 (2016)

6. Y.H. Kim, H. Cho, J.H. Heo, T.S. Kim, N.S. Myoung, C.L. Lee, S.H. Im, T.W. Lee, Adv. Mater. 27, 1248 (2015)

7. X. Hong, B. Zhang, E. Murphy, J. Zou, F. Kim, J. Power Sour. 343, 60 (2017)

8. L. Tang, F. Duan, M. Chen, RSC Adv. 6, 65012 (2016)

9. W. Tang, L. Peng, C. Yuan, J. Wang, S. Mo, C. Zhao, Y. Yu, Y. Min, A.J. Epstein, Synth. Met. 202, 140 (2015)

10. D.G. Papageorgiou, I.A. Kinloch, R.J. Young, Prog. Mater. Sci. 90, 75 (2017)

11. M.D.A. Khan, A. Akhtar, S.A. Nabi, New J. Chem. 39, 3728 (2015)

12. V. Ganesan, A. Jayaraman, Soft Matter 10, 13 (2014)

13. S.K. Kumar, N. Jouault, B. Benicewicz, T. Neely, Macromolecules 46, 3199 (2013)

14. S.K. Kumar, R. Krishnamoorti, Annu. Rev. Chem. Biomol. Eng. 1, 37 (2010)

15. L.M. Hall, A. Jayaraman, K.S. Schweizer, Curr. Opin. Solid State Mater. Sci. 14, 38 (2010)

16. R. Gangopadhyay, A. De, Chem. Mater. 12, 608 (2000)

17. S. Komarneni, J. Mater. Chem. 2, 1219 (1992)

18. P.C. Lebaron, Z. Wang, T.J. Pinnavaia, Appl. Clay Sci. 15, 11 (1999)

19. V. Mittal, Materials (Basel) 2, 992 (2009)

20. Y. Bréchet, J.Y.Y. Cavaillé, E. Chabert, L. Chazeau, R. Dendievel, L. Flandin, C. Gauthier, Adv. Eng. Mater. 3, 571 (2001)

21. M. Berggren, D. Nilsson, N.D. Robinson, Nat. Mater. 6, 3 (2007)

22. A.C. Arias, J.D. MacKenzie, I. McCulloch, J. Rivnay, A. Salleo, Chem. Rev. 110, 3 (2010)

23. L.L. Lavery, G.L. Whiting, A.C. Arias, Org. Electron. Phys. Mater. Appl. 12, 682 (2011)

24. H. Nagashima, R. Onody, R. Faria, Phys. Rev. B 59, 905 (1999)

25. B. Derrida, J. Vannimenus, J. Phys. A Math. Gen. 15, L557 (1982)

26. S. Miyata, Y. Sakuratani, X.T. Tao, Opt. Mater. (Amst) 21, 99 (2003)

27. Y. Cao, G.M. Treacy, P. Smith, A.J. Heeger, Synth. Met. 57, 3526 (1993)

28. Y. Yang, A.J. Heeger, Appl. Phys. Lett. 64, 1245 (1994)

29. J.C. Dyre, J. Appl. Phys. 64, 2456 (1988)

30. R.S. Kohlman, J. Joo, Y.Z. Wang, J.P. Pouget, H. Kaneko, T. Ishiguro, A.J. Epstein, Phys. Rev. Lett. 74, 773 (1995)

31. D. Stauffer, A. Aharony, Computer (Long. Beach. Calif) 1, 192 (1994)

32. S.A. Gordeyev, F.J. Macedo, J.A. Ferreira, F.W.J. Van Hattum, C.A. Bernardo, Phys. B 279, 33 (2000)

33. S.H. Foulger, J. Appl. Polym. Sci. 72, 1573 (1999)

34. F. Carmona, Phys. A Stat Mech. Appl. 157, 461 (1989)

35. C.-W. Nan, Y. Shen, J. Ma, Annu. Rev. Mater. Res. 40, 131 (2010)

36. S. Vionnet-Menot, C. Grimaldi, T. Maeder, S. Strässler, P. Ryser, Phys. Rev. B Condens. Matter Mater. Phys. 71, 1 (2005)

37. S. Xu, O. Rezvanian, K. Peters, M.A. Zikry, Nanotechnology 24 , 155706 (2013)

38. G. Ambrosetti, C. Grimaldi, I. Balberg, T. Maeder, A. Danani, P. Ryser, Phys. Rev. B Condens. Matter Mater. Phys. 81, 1 (2010) 
39. C.W. Nan, Prog. Mater. Sci. 37, 1 (1993)

40. D. Toker, D. Azulay, N. Shimoni, I. Balberg, O. Millo, Phys. Rev. B 68, 12 (2003)

41. Z. Rubin, S.A. Sunshine, M.B. Heaney, I. Bloom, I. Balberg, Phys. Rev. B 59, 12196 (1999)

42. L.H.C. Mattoso, R.M. Faria, L.O.S. Bulhões, A.G. MacDiarmid, J. Polym. Sci. Part A Polym. Chem. 32, 2147 (1994)

43. S.K. Manohar, A.G. Macdiarmid, A.J. Epstein, Synth. Met. 41, $711(1991)$

44. M.J. Da Silva, D.H.F. Kanda, H.N. Nagashima, J. Non. Cryst. Solids 358, 270 (2012)

45. B. Derrida, J.G. Zabolitzky, J. Vannimenus, D. Stauffer, J. Stat. Phys. 36, 31 (1984)

46. M.K. Simon, Probability Distributions Involving Gaussian Random Variables: A Handbook for Engineers and Scientists, 1st edn. (Springer, Boston, MA, 2006), pp. 1-23
47. R.F. Bianchi, G.F.L. Ferreira, C.M. Lepienski, R.M. Faria, I. Introduction, J. Chem. Phys. 110, 4602 (1999)

48. V.S. Machavariani, J. Phys. Condens. Mater. 13, 6797 (2001)

49. G. Ambrosetti, I. Balberg, C. Grimaldi, Phys. Rev. B Condens. Matter Mater. Phys. 82, 1 (2010)

50. I. Balberg, Phys. Rev. Lett. 59, 1305 (1987)

51. I. Balberg, Carbon N. Y. 40, 139 (2002)

52. R. Simoes, J. Silva, S. Lanceros-Mendez, R. Vaia, J. Mater. Sci. 45, 268 (2010)

53. R. Simoes, J. Silva, R. Vaia, V. Sencadas, P. Costa, J. Gomes, S. Lanceros-Méndez, Nanotechnology 20, 35703 (2009)

54. J. Frenkel, Phys. Rev. 54, 647 (1938)

55. J.G. Simmons, Phys. Rev. 155, 657 (1967) 